\title{
Interleukin-11 Up-Regulates Survivin Expression in Endothelial Cells through a Signal Transducer and Activator of Transcription-3 Pathway
}

\author{
Keyvan Mahboubi, Fengzhi Li, Janet Plescia, Nancy C. Kirkiles-Smith, Mehdi Mesri, \\ Yuefen Du, Joseph M. Carroll, Jack A. Elias, Dario C. Altieri, and Jordan S. Pober \\ Interdepartmental Program in Vascular Biology and Transplantation (KM, FL, JP, NCK-S, MM, DCA, JSP), Boyer \\ Center for Molecular Medicine, and Section of Pulmonary and Critical Care Medicine (YD, JAE), Yale University \\ School of Medicine, New Haven, Connecticut; and Genetics Institute Inc. (JMC), Andover, Massachusetts
}

\begin{abstract}
SUMMARY: Interleukin-11 (IL-11) reduces injury both in vivo and in vitro, but the mechanisms are unknown. Stimulation of serum- and growth factor-deprived HUVEC with IL-11 increased survivin mRNA and protein expression levels in a dosedependent manner, with maximal induction at 50 to $100 \mathrm{ng} / \mathrm{ml}$ of IL-11. Survivin mRNA expression peaked after 3 to 6 hours of IL-11 treatment and decreased by 24 hours. Survivin protein expression was maximal at 6 hours of treatment and remained elevated through 24 hours. Survivin induction may be mediated by activation of protein kinase B/Akt, but IL-11 failed to activate this pathway in HUVEC. IL-11 did activate signal transducer and activator of transcription (STAT)-3 and IL-11 failed to induce survivin expression in HUVEC transduced with a dominant-negative STAT3 mutant, whereas control-transduced HUVEC responded normally. An IL-11 transgene caused increased survivin mRNA expression in mice compared with control littermates. Intradermal injection of IL-11 (500 ng) into human skin xenografts on immunodeficient mice up-regulated survivin protein in microvascular endothelium and epithelial keratinocytes. We conclude that IL-11 induces expression of survivin, an antiapoptotic protein, in vitro and in vivo, and identify STAT3 as a critical mediator of this response. (Lab Invest 2001, 81:327-334).
\end{abstract}

\begin{abstract}
$\Gamma$ nter nterleukin-11 (IL-11) is an interleukin-6 (IL-6)-type cytokine that interacts with a variety of hematopoietic and nonhematopoietic cell types. IL-11 has been shown to have protective effects in various in vivo models of injury, both in the gastrointestinal tract (Du et al, 1997; Keith et al, 1994; Orazi et al, 1996) and in the lung (Waxman et al, 1998). Human IL-11, expressed as a transgene in bronchial mucosa, markedly diminish hyperoxic lung injury in mice (Waxman et al, 1998). This effect was associated with a reduction in multiple parameters of lung injury, including alveolarcapillary protein leak, endothelial cell and epithelial cell membrane injury, lipid peroxidation, pulmonary neutrophil recruitment, inflammatory cytokine production, and DNA fragmentation (Waxman et al, 1998). The protective effects of IL-11 are not restricted to in vivo models of injury. Recently, we reported that pretreatment of cultured HUVEC with IL-11 can protect these cells from immune-mediated injury due to cytotoxic T lymphocyte or antibody (Ab) plus complement (Mahboubi et al, 2000). In vitro cytoprotection by
\end{abstract}

Received October 11, 2000.

This work was supported by National Institutes of Health grants F32HL10275 (to KM); RO1-HL36708 and P50-HL56389 (to JAE); RO1HL54131 and RO1-CA78810 (to DCA); and HL36003 and HL62188 (to JSP).

Address reprint requests to: Dr. Jordan S. Pober, Boyer Center for Molecular Medicine, Room 454, 295 Congress Ave, New Haven, CT 06510. E-mail: jordan.pober@yale.edu
IL-11 is dependent on new protein synthesis (Mahboubi et al, 2000). Although it is likely that induction of specific gene products is required for cytoprotective effects of IL-11 in vitro, the exact mechanisms of cytoprotection or the identities of such putative antiapoptotic proteins are not known.

IL-11 activates cells through a multimeric receptor containing a signal-transducing subunit, gp130, and a unique ligand binding subunit, IL-11-receptor- $\alpha$ (IL$11 \mathrm{R} \alpha$ ) chain (Heinrich et al, 1998). Binding of IL-11 to IL-11R $\alpha$ induces clustering of gp130, leading to phosphorylation of signal transducer and activator of transcription (STAT)-3 as well as p42 and p44 mitogenactivated protein kinases (MAPK) at concentrations of $\mathrm{IL}-11$ as low as $1 \mathrm{ng} / \mathrm{ml}$ (Mahboubi et al, 2000). STAT1 is also activated by IL-11, but only at concentrations of IL-11 above $10 \mathrm{ng} / \mathrm{ml}$ (Mahboubi et al, 2000). Recently, phosphotidylinositol-3 kinase (PI-3 kinase) has been described to be activated by IL- 6 and certain other cytokines that use gp130 (Chung et al, 2000; Oh et al, 1998; Qiu et al, 1998). Activation of the PI-3 kinase pathway results in the phosphorylation and subsequent activation of an antiapoptotic serine/threonine kinase Akt (also known as protein kinase B) (Hemmings, 1997).

Survivin is a member of the inhibitor of apoptosis protein family that is highly expressed in many human cancers, but is undetectable in normal adult tissues in vivo (Ambrosini et al, 1997; Reed and Reed, 1999). Survivin expression can be up-regulated in cultured 
endothelial cells (EC) either by growth factors, such as vascular endothelial cell growth factor (VEGF), in a cell cycle-dependent manner (O'Connor et al, 2000; Tran et al, 1999) or by the vascular remodeling factor angiopoietin-1 in a cell cycle-independent manner (Papapetropoulos et al, 2000). In vivo, survivin expression is observed in untransformed EC that are involved in an angiogenic response (O'Connor et al, 2000), ie, in a setting where EC are exposed to VEGF and angiopoietin-1. The angiopoietin effect is mediated via the PI-3 kinase/Akt pathway (Papapetropoulos et al, 2000). Overexpression of survivin can reduce apoptosis induced either by growth factor withdrawal or by TNF/cycloheximide in cultured EC (O'Connor et al, 2000). These observations have led to the hypothesis that a physiologic role of survivin is to protect angiogenic endothelium from apoptosis.

We report here that IL-11 induces survivin expression in HUVEC in vitro and in vivo xenograft and transgenic models in vivo. This pathway appears independent of Akt signaling because IL-11 does not activate this enzyme in HUVEC. In contrast, we find that this response is dependent upon signaling through STAT3.

\section{Results}

\section{IL-11 Increases Survivin mRNA and Protein Levels in Cultured HUVEC}

We examined the effects of IL-11 on survivin expression in cultured HUVEC. Under standard culture conditions, ie, in the presence of $20 \%$ FCS and $50 \mu \mathrm{g} / \mathrm{ml}$ of fibroblast growth factor-1 (FGF-1), survivin mRNA and protein were basally expressed in HUVEC. The levels declined as the cells were made quiescent by serum and growth factor withdrawal for 36 to 48 hours (Fig. 1, control). Treatment of quiescent cells with IL-11 caused a time-dependent increase in survivin mRNA levels above untreated cells (Fig. 1), which peaked 1 to 3 hours after stimulation and remained sustained up to 24 hours (Fig. 1). Preincubation of

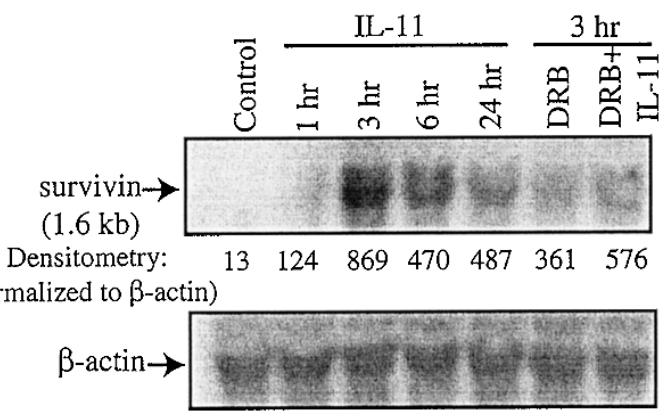

Figure 1.

Time-dependent induction of survivin mRNA by IL-11 in HUVEC. Confluent HUVEC were made quiescent by culture in media containing $0.1 \%$ FCS for 36 hours, after which new media without (control) or with IL-11 (100 ng/ml) was added at the indicated time intervals. Survivin and $\beta$-actin RNA were examined by Northern hybridization. In the indicated samples, 5, 6, dichlorobenzimidazole riboside (DRB; $20 \mu \mathrm{m}$ ) was added 30 minutes before the addition of IL-11. Numbers below the survivin panel indicate relative levels based on densitometry. Data are from one of two experiments with similar outcome. cells with the transcriptional inhibitor, 5, 6, dichlorobenzimidazole riboside (DRB) $(20 \mu \mathrm{M})$ blocked upregulation of survivin induced by IL-11 (Fig. 1). These data suggest that IL-11 increases survivin mRNA by increasing transcription of the survivin gene.

Consistent with the expected molecular weight of survivin, an affinity-purified rabbit polyclonal $\mathrm{Ab}$ to survivin immunoblotted a single band of about 16.5 kDa from HUVEC (Fig. 2). Expression of endogenous survivin protein in quiescent HUVEC fell to levels that were minimally detectable by immunoblotting 36 to 48 hours after serum and growth factor were withdrawn (Fig. 2A, control). IL-11 induced the expression of survivin protein in quiescent HUVEC in a timedependent manner, which peaked approximately 6 hours after stimulation and remained elevated for up to 24 hours (Fig. 2A). Survivin induction by IL-11 was concentration-dependent, showing a maximal effect at 50 to $100 \mathrm{ng} / \mathrm{ml}$ (Fig. 2B).

\section{IL-11 Does Not Induce Serine Phosphorylation of Akt in HUVEC}

Induction of survivin expression by angiopoietin-1 in EC is mediated by $\mathrm{PI}-3$ kinase/Akt-dependent pathway (Papapetropoulos et al, 2000). To assess the activation of Akt in HUVEC we tested whether IL-11 treatment results in phosphorylation of Akt as detected by immunoblotting using a serine 437 phospho-Akt-specific Ab. Stimulation of HUVEC with either 100 or $500 \mathrm{ng} / \mathrm{ml}$ of IL-11 did not induce Akt phos-
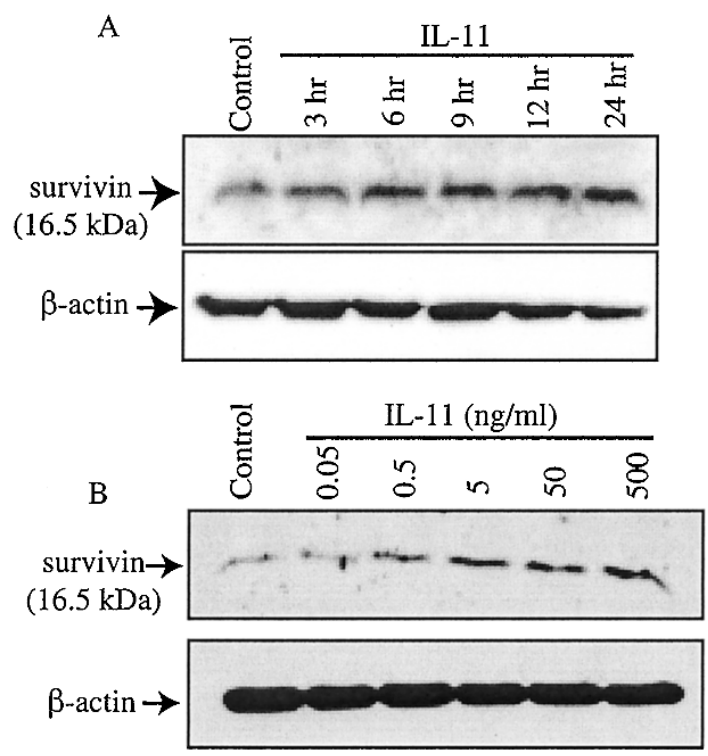

Figure 2.

IL-11 induces survivin protein expression in HUVEC. A, Time-dependent expression of survivin protein. The experimental conditions are the same as for Figure 1. At the indicated treatment intervals, quiescent HUVEC were harvested and analyzed for survivin and $\beta$-actin protein expression using immunoblotting as described in "Materials and Methods." B, IL-11 dose-dependent expression of survivin protein. HUVEC were made quiescent as described in Figure 1 and stimulated with various concentrations of IL-11. After 12 hours of treatment, cells were harvested and analyzed for survivin and $\beta$-actin using immunoblotting as described in "Materials and Methods." For both A and B, data are from one of three experiments with similar outcome. 
phorylation (Fig. 3). However, IL-11 was active because it clearly increased phosphorylation of p42/p44 MAPK in these cells (Fig. 3). IL-11 at concentrations less than $100 \mathrm{ng} / \mathrm{ml}$ was also unable to cause phosphorylation of Akt (data not shown). Our previous studies had shown that oncostatin $M$ is a much stronger activator of gp130 signaling responses in HUVEC than is IL-11 (Mahboubi et al, 2000). Stimulation of HUVEC with oncostatin M did increase Akt phosphorylation in a dose-dependent manner (Fig. 3). These data demonstrate that gp130 signals in HUVEC can result in Akt activation, but that this requires a stronger signal than that provided by IL-11.

\section{Down-Negative STAT3 Inhibits IL-11-Induced Survivin Expression in HUVEC}

IL-11 activates STAT3 in HUVEC (Mahboubi et al, 2000). Therefore, we investigated the functional role of STAT3 in IL-11-induced survivin expression in these cells. For this purpose, we stably transduced HUVEC with retroviruses encoding either enhanced green fluorescent protein (EGFP), STAT3(Y705F)-FLAG, or STAT1(Y701F)-hemagglutinin (HA). The M2 monoclonal anti-FLAG Ab and anti-STAT3 Ab were used to confirm expression of STAT3(Y705F)-FLAG in transduced cells with this gene (Fig. 4A), whereas a monoclonal $\mathrm{Ab}$ to HA or STAT1 Ab was used to demonstrate the expression of STAT1(Y701F)-HA in transduced cells (Fig. 4B). STAT3-FLAG and STAT1-HA were only detected in cells transduced with STAT3(Y705F)-FLAG gene (Fig. 4A, upper panel) and STAT1(Y701F)-HA (Fig. 4B, upper panel), respectively. Single protein bands were detected in cells transduced with EGFP gene corresponding to endogenous levels of STAT3 and STAT1 protein by using antibodies to STAT3 and STAT1, respectively (Fig. 4, A and B, lower panels). This contrasts with the appearance of an additional protein band of slightly higher molecular weight detected in cells transduced with either STAT3(Y705F)-FLAG gene or STAT1(Y705F)-HA gene (Fig. 4B, lower panel).

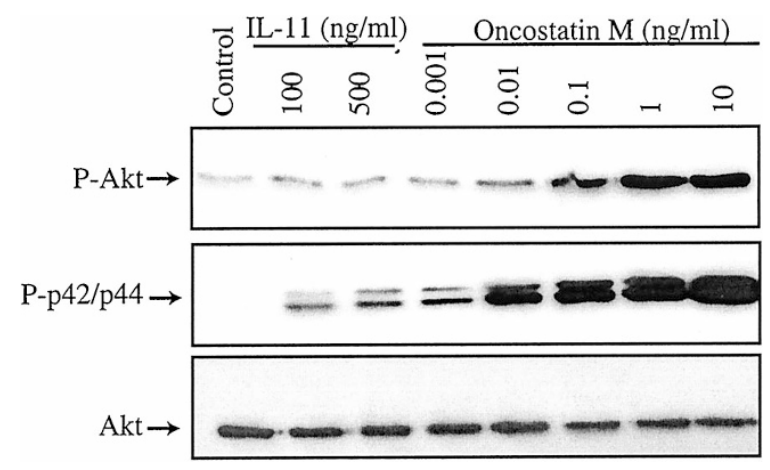

Figure 3.

IL-11 does not stimulate Akt phosphorylation in HUVEC. Quiescent HUVEC were incubated with various doses of IL-11 and oncostatin M (positive control) for 15 minutes and analyzed for either Akt phosphorylation (P-Akt, ser ${ }^{473}$ upper panel), p42/p44 MAPK phosphorylation (middle panel, P-p42/p44, $\mathrm{Thr}^{202} / \mathrm{Tyr}^{204}$ ), or total Akt (lower panel) by immunoblotting as described in the "Materials and Methods." Data are from one of two experiments with similar outcome.
We next investigated the tyrosine phosphorylation of endogenous STAT3 in EGFP, or STAT3(Y705F)FLAG-transduced HUVEC. To do this, the cells were stimulated with various doses of IL-11 (0.5 to 500 $\mathrm{ng} / \mathrm{ml}$ ) for 10 minutes and the cell lysates were analyzed by immunoblot using specific P-STAT3 Ab. IL-11 dose-dependently induced tyrosine phosphorylation of STAT3 in EGFP-transduced HUVEC, in agreement with our previous observations using untransduced HUVEC (Fig. 5) (Mahboubi et al, 2000). The concentration of IL-11 required to cause tyrosine phosphorylation of endogenous STAT3 protein was increased by about 100-fold in STAT3(Y705F)-FLAG-transduced cells (Fig. 6), confirming that this mutation acts as a dominant negative (dn). Parallel experiments were conducted in STAT1(Y705F)-HA-transduced HUVEC. Despite the fact that STAT1(Y701F)-HA protein was expressed in HUVEC at roughly similar levels as STAT3(Y705F)-FLAG (Fig. 4B), neither IL-11-mediated tyrosine phosphorylation of endogenous STAT1 nor IL-11-mediated induction of survivin was abrogated by dn-STAT1 in these cells (data not shown).

Finally, we examined the effect of expression of dn-STAT3 protein on the IL-11-mediated induction of survivin protein. IL-11 significantly increased survivin expression in EGFP-transduced HUVEC (Fig. 6). In contrast, IL-11 stimulation of STAT3(Y705F)-FLAGtransduced cells did not result in up-regulation of
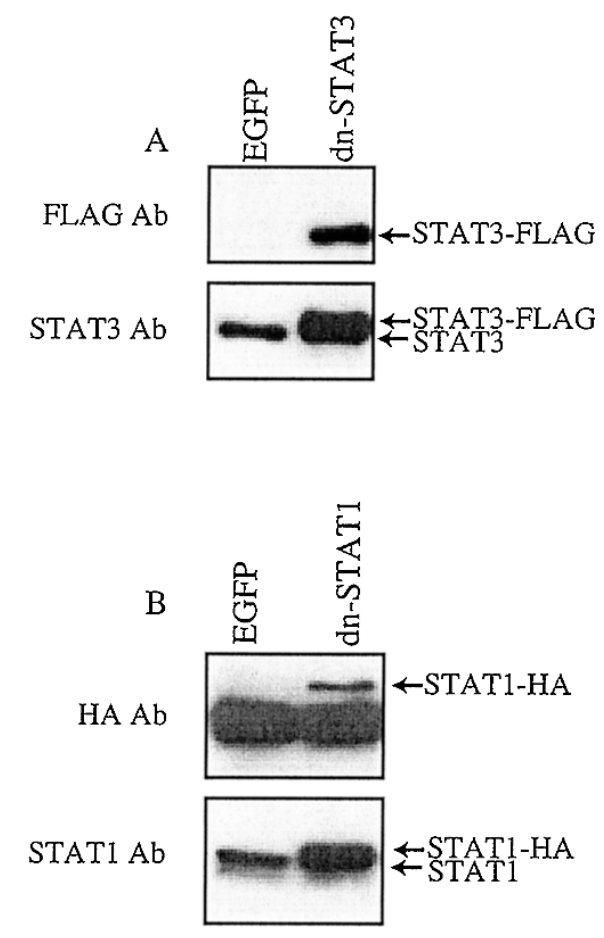

Figure 4.

Overexpression of dn-STAT3 and dn-STAT1 in HUVEC. HUVEC were transduced by retroviruses encoding either EGFP, dn-STAT3 (STAT3[Y705F]-FLAG), or dn-STAT1 (STAT1[Y701F]-HA) as described in "Materials and Methods." Cell lysates were analyzed for either STAT3-FLAG (Fig. 6A, upper panel), total STAT3 (Fig. 6B, lower panel), STAT1-HA (Fig. 6B, upper panel), or total STAT1 (Fig. 6B, lower panel) by immunoblotting as described in "Materials and Methods." Data are from one of two independent sets of transductants with similar outcome. 


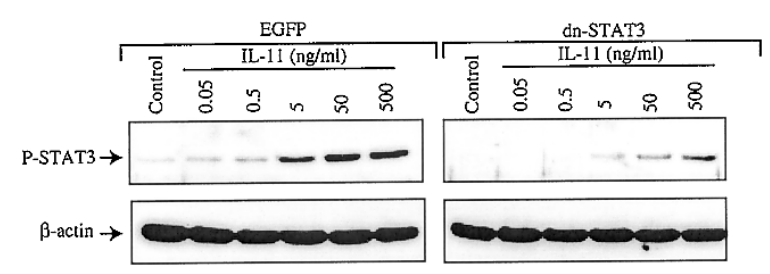

Figure 5.

Effect of dn-STAT3 on phosphorylation of tyrosine 705 of endogenous STAT3 by IL-11. HUVEC were transduced by retroviruses encoding either EGFP or dn-STAT3 (STAT3[Y705F]-FLAG) and stimulated with various concentrations of IL-11 for 10 minutes. Cell lysates were analyzed for either STAT3 phosphorylation (P-STAT3, Tyr ${ }^{705}$, upper panel) or $\beta$-actin (lower panel) by immunoblotting as described in "Materials and Methods." Data are from one of two independent sets of transductants with similar outcome.

survivin (Fig. 6). These data indicate that IL-11 mediates up-regulation of survivin through a STAT3dependent pathway.

\section{IL-11 Up-Regulates Survivin mRNA and Protein In Vivo}

Mice transgenic for human $\mathrm{IL}-11$ regulated by the CC10 promoter express this protein at high levels in the lung (Ray et al, 1997). We performed Northern hybridization to compare the levels of survivin mRNA in the lungs of IL-11 transgene $(+)$ and IL-11 transgene (-) control littermates. In Northern blots, a survivinspecific single-strand probe detected a predominant 1.9-kb transcript (Fig. 7, upper panel). Higher levels of survivin transcript were detected in IL-11 transgene (+) animals than in littermate controls (Fig. 7, upper panel). The amount of $28 \mathrm{~S}$ and $18 \mathrm{~S}$ ribosomal RNA confirmed comparable loading of mRNA per sample (Fig. 7, lower panel). These data indicate that survivin gene expression is induced by IL-11 in vivo.

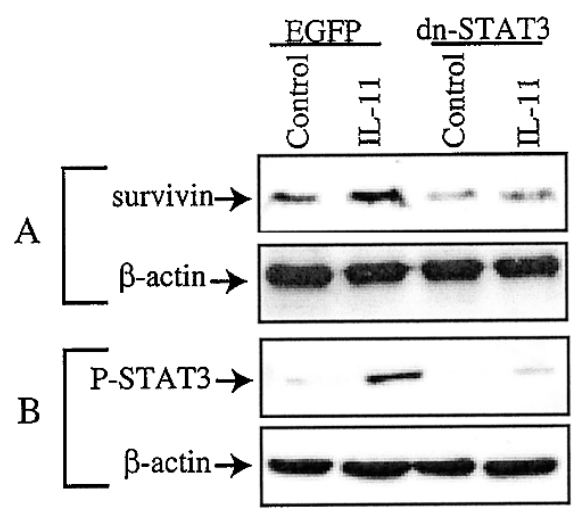

Figure 6 .

Effect of overexpression of dn-STAT3 protein on induction of survivin by IL-11. HUVEC were transduced by retroviruses encoding either EGFP or dn-STAT3 (STAT3[Y705F]-FLAG). A, Transduced cells were made quiescent as described in Figure 2 and stimulated with IL-11 (5 ng/ml) for 12 hours and analyzed for either survivin (upper panel) or $\beta$-actin (lower panel) protein by immunoblotting as described in "Materials and Methods." B, Transduced HUVEC were stimulated with IL-11 $(5 \mathrm{ng} / \mathrm{ml})$ for 10 minutes and analyzed for either STAT3 phosphorylation (P-STAT3, Tyr ${ }^{705}$, upper panel) or $\beta$-actin (lower panel) by immunoblotting as described in "Materials and Methods." Data are from one of three experiments involving two independent sets of transductants with similar outcome.

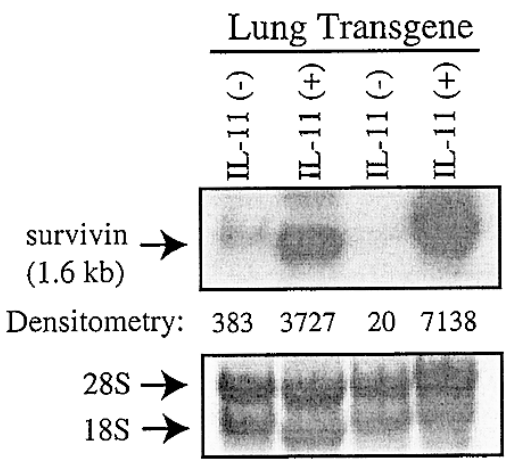

Figure 7

Survivin mRNA in lungs of CC-10-IL-11 transgene $(+)$ and transgene $(-)$ mice. Total lung mRNA was isolated and analyzed by Northern blotting as described in "Materials and Methods" (upper panel). The amount of $28 \mathrm{~S}$ and 18S RNA on the Nylon filters was detected by methylene blue staining and was used for comparison of sample loading (lower panel). Numbers below the survivin panel indicate relative levels based on densitometry. Northern blots of two pairs of animals out of four pairs examined, all with similar results, are shown.

To further establish whether IL-11 could increase survivin protein in vivo, we used a human skin xenograft model developed in severe combined immunodeficient (SCID)/beige mice (Murray et al, 1998). Immunostaining of human skin grafts with survivin $A b$ demonstrated that intradermal injection of IL-11 into human skin grafts increased survivin protein expression in these grafts (Fig. 8, panel A vs panel B). Up-regulation of survivin was detected both in the epidermal keratinocytes and in dermal microvascular endothelium. These data establish that IL-11 can increase survivin protein expression in vivo.

\section{Discussion}

In this report we have shown that IL-11 up-regulates survivin expression in vitro and in vivo. The induction of survivin mRNA by $\mathrm{IL}-11$ in endothelial cells is mediated, at least in part, by increasing transcription of the survivin gene. IL-11 stimulates survivin expression in EC via a STAT3-dependent pathway.

Previous studies have demonstrated that survivin is up-regulated in endothelial cells by mediators of angiogenesis such as VEGF, FGF, and angiopoietin (O'Connor et al, 2000; Papapetropoulos et al, 2000; Tran et al, 1999). However, survivin is not regulated by proinflammatory cytokines such as interleukin-1 and TNF (O'Connor et al, 2000). IL-11 is not a mitogen for EC nor does it regulate the inflammatory functions of EC either positively or negatively. Although it shares with angiogenic factors the capacity to induce survivin, IL-11 seems to do so via a different signaling pathway because it does not activate Akt. Previous studies demonstrated that both overexpression of dn-STAT3 and dn-Akt inhibited the antiapoptotic effects of IL-6, another gp130-signaling cytokine, against transforming growth factor- $\beta$-induced apoptosis in human hepatoma cell line (Chen et al, 1999). Similarly, overexpression of dn-STAT3 completely abolishes gp130-mediated survival signals in a pro-B 

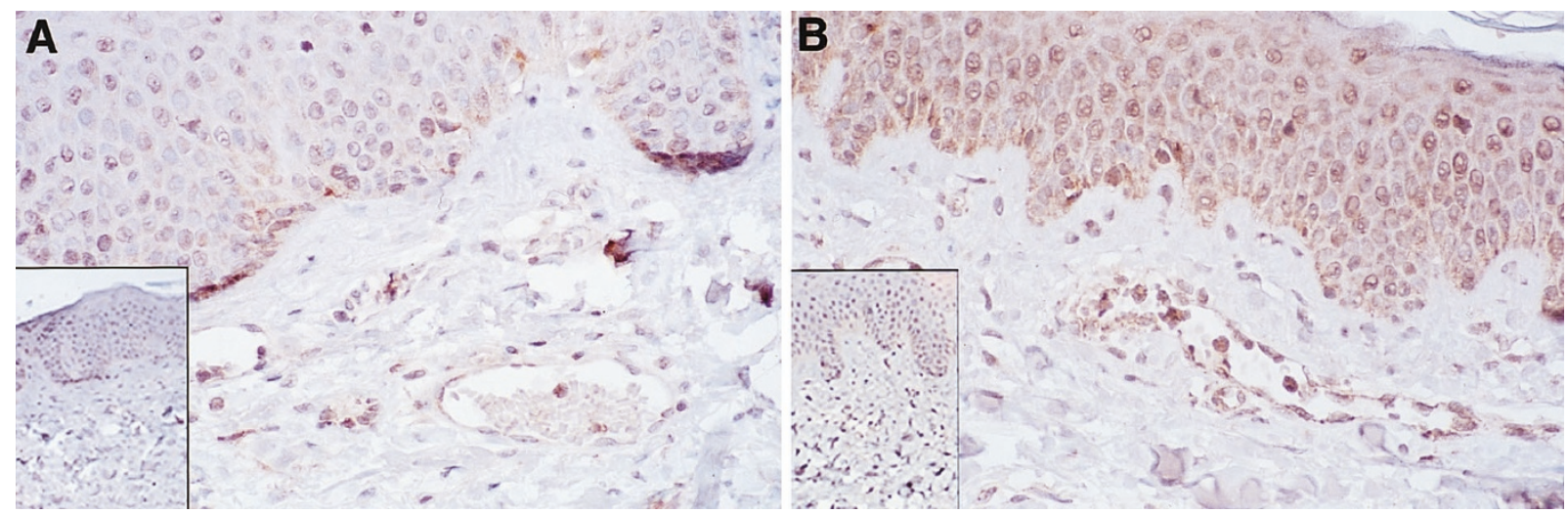

Figure 8.

Representative photomicrographs (original magnification, $\times 40$ ) of human skin grafts demonstrating the IL-11 up-regulated survivin protein expression in vivo. Human skin grafts on SCID/beige mice were harvested 24 hours after interdermal injection of either saline control (A) or $500 \mathrm{ng}$ of IL-11 (B). Skin grafts were immunostained with either control Ab (A and B, insets) or antisurvivin Ab ( $A$ and B) as described in "Materials and Methods." Similar findings were observed in three additional pairs of animals providing two independent experiments.

cell line (Fukada et al, 1996). These results imply that both STAT3 and PI-3 kinase/Akt pathways are important for gp130-mediated antiapoptotic signals (Chen et al, 1999; Chung et al, 2000; Fukada et al, 1996).

The targets of STAT3 activation are not fully understood. STAT3 activation via gp130 increases expression of $\mathrm{Bcl}-2$, an antiapoptotic protein, in pro-B cells (Fukada et al, 1996). Therefore, up-regulation of Bcl-2 is considered as one of the antiapoptotic mechanisms of STAT3. In addition, overexpression of STAT3 in osteoblastic cells potentiated both IL-6- and oncostatin M-mediated induction of p21 (Cip1, also known as WAF1, sd11) which also protects against apoptosis (Bellido et al, 1998). Although IL-11 activates STAT3 in HUVEC, IL-11 did not increase the expression of $\mathrm{Bcl}-2$ or p21 (unpublished observations, KM) in these cells. These data are consistent with the idea that STAT3 may induce the expression of a different set of genes, depending on the cell type involved.

IL-11 significantly reduced epithelial and endothelial cell membrane injury associated with hyperoxia in the lung (Waxman et al, 1998). Cell death responses with features of necrosis and apoptosis play important roles in the pathogenesis of hyperoxic lung injury (Mantell et al, 1999). Interestingly, IL-11 diminished DNA fragmentation, a marker of apoptosis and necrosis, in the lung of mice exposed to $100 \% \mathrm{O}_{2}$ (Waxman et al, 1998). The exact mechanisms by which IL-11 decreases apoptosis in vivo are not clearly understood. An interesting finding of our study is the demonstration that IL-11 (+) transgenic mice express higher survivin mRNA levels and that administration of $\mathrm{IL}-11$ induced de nova survivin expression in human skin xenografts. Thus, survivin protein expression may contribute to these effects.

A major question raised by the present study is to what extent survivin induction contributes to the cytoprotective actions of IL-11 we have previously reported in HUVEC. In in vitro experiments, IL-11 exerted a cytoprotective action on HUVEC cultured in complete media (ie, media containing serum and FGF-1) (Mahboubi et al, 2000), conditions under which survivin is already expressed before the addition of IL-11. In this setting, it is therefore likely that IL-11 activates additional protective responses. We are unable to assess the protective effects of IL-11 in quiescent HUVEC because, although these cells are useful for evaluation of gene expression, they are extremely fragile and not suited for evaluation of cytoprotection. In vivo, survivin is not basally expressed in endothelium, and the induction of survivin in EC by IL- 11 could contribute to cytoprotection. In this context it is intriguing that in addition to canonical pathways of cell cycle progression, survivin induction can be triggered by nonmitogenic stimuli, including angiopoietin (Papapetropoulos et al, 2000) or IL-11 (this study). Although survivin expression in proliferating cells has been linked to both the control of apoptosis and prevention of ploidy ( $\mathrm{Li}$ et al, 1999), the presence of survivin in IL-11-stimulated, noncycling endothelium suggests a preferential role in cytoprotection, independently of cytokinesis. This question may be addressed by future studies using the mouse lung or human skin models described in this report.

Finally we have established a linkage between STAT3 and survivin gene activation. This result implies that other growth factor receptors, or nonreceptor type tyrosine kinases, or oncogene products that are capable of activating STAT3 could also induce survivin expression. This may have more general implications for the overexpression of survivin in cancer, where it constitutes the top fourth "transcriptome" in the most common human neoplasia (Velculescu et al, 1999). Recent data demonstrated that a constitutively active form of STAT3 was sufficient to produce anchorageindependent cell proliferation and tumor formation in nude mice, thus emphasizing its oncogenetic potential (Bromberg et al, 1999). The dramatic up-regulation of survivin expression via STAT3 activation reported here suggests that this pathway may be also operative during oncogenic transformation, further contributing to dysregulation of the apoptosis in cancer. The generality of the survivin-STAT3 pathway and its conse- 
quences for vascular responses and tumor growth warrant further exploration.

\section{Materials and Methods}

\section{Reagents, Cells, and Animals}

Recombinant human IL-11 was provided by Genetics Institute (Andover, Massachusetts). Recombinant human oncostatin $M$ was purchased from R\&D Systems (Minneapolis, Minnesota). FGF-1, commonly called endothelial cell growth supplement, was obtained from Collaborative Research/Becton Dickinson (Bedford, Massachusetts) and used in conjunction with porcine intestinal heparin (Sigma, St. Louis, Missouri). The transcriptional inhibitor, DRB, polybrene, and puromycin were obtained from Sigma. Mouse monoclonal $\mathrm{Ab}$ to $\beta$-actin and M2 monoclonal anti-FLAG Ab were obtained from Sigma. Rabbit polyclonal Ab reactive to STAT3, phosphotyrosine-STAT3, STAT1, phosphotyrosine STAT1, Akt, phosphoserine Akt, and phosphothreonine/phosphotyrosine-p42/p44 MAPK were purchased from New England Biolabs (Beverly, Massachusetts). Rabbit polyclonal Ab to survivin was made against recombinant survivin protein as described previously, and affinity purified (Grossman et al, 1999). Mouse monoclonal anti-HA Ab was purchased from Boehringer Mannheim (Indianapolis, Indiana).

Human EC were isolated from umbilical veins as previously described (Gimbrone, 1976; Thornton et al, 1983) and cultured on gelatin (J. T. Baker, Phillipsburg, New Jersey)-coated tissue culture plastic at $37^{\circ} \mathrm{C}$ in $5 \% \mathrm{CO}_{2}$-humidified air in Medium 199 containing 20\% FCS, 2 mm L-glutamine, $100 \mathrm{U} / \mathrm{ml}$ penicillin, $100 \mu \mathrm{g} / \mathrm{ml}$ streptomycin (all from Life Technologies, Grand Island, New York), $50 \mu \mathrm{g} / \mathrm{ml} \mathrm{FGF-1,} \mathrm{and} 100 \mu \mathrm{g} / \mathrm{ml}$ porcine intestinal heparin (Sigma). The PhoenixAmpho packaging cell line was obtained from Dr. G. Nolan (Stanford University, Stanford, California) and cultured in DMEM media containing 10\% FCS 2 mM L-glutamine, $100 \mathrm{U} / \mathrm{ml}$ penicillin, and $100 \mu \mathrm{g} / \mathrm{ml}$ streptomycin.

CC-10-IL-11 transgenic mice that overexpress IL-11 in a lung-specific fashion, were produced on CBA/C57BL6 background mice as described previously (Waxman et al, 1998). C.B-17 SCID/beige mice (Taconic Farms, Germantown, New York) were used at 5 to 8 weeks of age. The animals were housed in microisolator cages and were fed sterilized food and water.

\section{RNA Extraction and Northern Blotting}

To extract RNA from HUVEC, cells were lysed directly in a culture dish with TRI Reagent $\left(0.2 \mathrm{ml} / 10^{6}\right.$ cells $)$. For RNA extraction from mouse lung, the animals were anesthetized under a protocol approved by the Yale Animal Care and Use Committee. The lungs were perfused with PBS $(\mathrm{pH}$ 7.4) in situ, removed from the animal, and homogenized in TRI Reagent (Molecular Research Center, Cincinnati, Ohio) according to the manufacturer's specifications. For Northern blot anal- ysis, 10 to $20 \mu \mathrm{g}$ of total RNA was separated on $1 \%$ agarose gels with $7 \%$ formaldehyde, transferred to Nylon filters (Hybond-N; Amersham Pharmacia Biotech, Piscataway, New Jersey), ultraviolet cross-linked (Stratagene, La Jolla, California) and hybridized with either ${ }^{32} \mathrm{P}$-labeled survivin cDNA probe (Ambrosini et al, 1997) or ${ }^{32} \mathrm{P}$-labeled $\beta$-actin cDNA probe (Clontech, Palo Alto, California) in ExpressHyb hybridization solution (Clontech). After washing, the filter was exposed for autoradiography. Autoradiograms were scanned by using a laser densitometer (Fast Scan, Series 300; Molecular Dynamics, Sunnyvale, California).

\section{Immunoblotting}

Cells were washed twice with ice-cold PBS containing $1 \mathrm{~mm}$ sodium orthovanadate and $1 \mathrm{~mm}$ sodium fluoride and lysed with ice-cold RIPA lysis buffer (PBS, 1\% NP40, 0.5\% sodium deoxycholate, 0.1\% SDS, $1 \mathrm{~mm}$ PMSF, $10 \mu \mathrm{g} / \mathrm{ml}$ leupeptin, $1 \mathrm{~mm}$ sodium orthovanadate). Cell lysates were clarified by centrifugation at $10,000 \times g$ for 15 minutes, and protein concentrations of supernatant were determined by using a Bio-Rad assay kit (Bio-Rad, Hercules, California). Lysates were prepared for SDS-PAGE by adding an equal volume of 2X SDS-PAGE sample buffer (100 mm Tris-Cl, pH 6.8, $200 \mathrm{~mm}$ dithiothreitol, 4\% SDS, 0.2\% bromphenol blue, $20 \%$ glycerol) and heating the mixture in a boiling water bath for 3 minutes. Twenty micrograms of protein was separated on SDS-polyacrylamide gel electrophoresis and transferred onto a polyvinylidene difluoride membrane by electrophoresis (Immobilon P; Millipore, Bedford, Massachusetts). After blocking with Tris-buffered saline Tween (10 mM Tris- $\mathrm{HCl}$, $\mathrm{pH}$ 8.0, $0.150 \mathrm{~mm} \mathrm{NaCl}, 0.05 \%$ Tween 20 ) containing 5\% milk for 1 hour at room temperature, the membranes were incubated overnight at $4^{\circ} \mathrm{C}$ with blocking solution containing the indicated Ab. Membranes were washed and incubated with a suitable horseradish peroxidase (HRP)-conjugated detecting reagent (Jackson Immuno Research, West Grove, Pennsylvania) and HRP activity was detected using an enhanced chemiluminescence kit, according to the manufacturer's instructions (Pierce, Rockford, Illinois). Autoradiograms were scanned using a laser densitometer (Fast Scan, Series 300; Molecular Dynamics, Sunnyvale, California).

\section{Construction of Retroviral Vectors Expressing EGFP, dn-STAT3, or dn-STAT1 and Stable Transduction of dn-STAT3, dn-STAT1, or EGFP}

dn-STAT3 and dn-STAT1 were made by replacing tyrosine 705 and 701 , in the respective wild type sequences, with phenylalanine (Kaptein et al, 1996; Walter et al, 1997). A C-terminal FLAG-tagged version of human dn-STAT3 [STAT3(Y705F)-FLAG] cDNA in expression vector pSL1180 was obtained from Dr. X. Y. Fu (Department of Pathology, Yale University School of Medicine), and the cDNA insert (2400 bp) was isolated by PCR and subcloned into the 
LZRSpBMN-Z retroviral vector (kindly provided by Dr. A. L. M. Bothwell, Section of Immunobiology, Yale University School of Medicine) by using 5 ' Hindlll and $3^{\prime}$ Notl cloning sites. An N-terminal HA-tagged version of human dn-STAT1 (STAT1[Y701F]-HA) cDNA in expression vector pSG91 was also obtained from Dr. X. Y. Fu and the cDNA insert (2400 bp) was isolated by $P C R$ and subcloned into the LZRSpBMN-Z retroviral vector using 5' BamHI and 3' Notl cloning sites. LZRSpBMN-Z retroviral vector DNA containing EGFP was kindly provided from Dr. A. L. M. Bothwell (Zheng et al, 2000). These retroviral vectors containing EGFP, (STAT3[Y705F]-FLAG), or (STAT1[Y701F]-HA) were directly transfected into the Phoenix-Amphoteric packaging cell line using lipofectamine plus reagent (Life Technologies). Two days after transfection, Phoenix cells were selected in the media containing puromycin (1 $\mu \mathrm{g} / \mathrm{ml}$ ). Puromycin-resistant cells were used to condition medium, providing a source of retroviral stock.

Infection of HUVEC was accomplished by serial infections over two weeks, without drug selection, as previously described by Zheng et al (2000). A round of viral infections in the presence of polybrene $(8 \mu \mathrm{g} / \mathrm{ml})$ was performed for 5 hours with HUVEC at passage one. The normal growth medium was replaced and cells were maintained overnight. The infection was repeated the next day. Cells were carried in culture for one week and then the process of double infection was repeated. Using this protocol the percentage of HUVEC-expressing transduced genes was more than $95 \%$.

\section{Skin Grafting}

Discarded normal adult human abdominal skin was obtained through the Yale Medical School Department of Pathology under protocol approved by the Human Investigation Committee. Human skin was transplanted to SCID/beige mice as previously described under a protocol approved by Yale Animal Care and Use Committee (Murray et al, 1998). In brief, superficial portions of the skin were harvested in $0.5-\mathrm{mm}$ thick sheets using a Goulian dermatome knife, gauge size, 0.016 (Weck, Research Triangle Park, North Carolina), and cut into approximately $1 \mathrm{~cm}^{2}$ pieces, which were kept in RPMI 1640 (Life Technologies) at $4^{\circ} \mathrm{C}$ until transplantation (not longer than 6 hours). SCID/beige mice were anesthetized by inhalation of methoxyfluride (Pitman-More, Mundelein, Illinois). The skin was shaved, two skin segments of equal size to human grafts were excised from the back of each mouse. The skin reproducibly engrafted with a more than $95 \%$ success rate and was allowed to heal for 5 weeks. IL-11 (500 ng/20 $\mu$ l of saline) or vehicle control (20 $\mu$ l of saline) was injected intradermally, Twentyfour hours later, the animals were killed under anesthesia and the skin grafts were removed and prepared for immunohistochemistry, as described below.

\section{Immunohistochemistry}

Each human skin graft was fixed in buffered formalin and used to prepare $3-\mu \mathrm{m}$ paraffin-embedded sections. Sections were deparaffinized in xylene and rehydrated in graded alcohol with quenching of endogenous peroxidase activity by treatment with $2 \%$ $\mathrm{H}_{2} \mathrm{O}_{2}$ in methanol. For immunostaining, the slides were boiled for 5 minutes in a standard pressure cooker, blocked in $10 \%$ normal goat serum, and incubated with either affinity-purified rabbit antisurvivin $A b$ or rabbit lgG $(0.5 \mu \mathrm{g} / \mathrm{ml})$ for 24 hours at $4^{\circ} \mathrm{C}$. After washes, the slides were incubated with biotinconjugated goat antirabbit IgG (Vector Laboratory, Burlingame, California) for 30 minutes at $22^{\circ} \mathrm{C}$, followed by streptavidin-conjugated peroxidase (Zymed Laboratory, San Francisco, California) and 3', 3'diaminobenzidine (Zymed Laboratory). Sections were counterstained with hematoxylin (Sigma).

\section{Acknowledgements}

We thank Louise Benson, Gwen Davis, and Lisa Gras for excellent technical assistance in cell culture.

\section{References}

Ambrosini G, Adida C, and Altieri DC (1997). A novel antiapoptosis gene, survivin, expressed in cancer and lymphoma. Nat Med 3:917-921.

Bellido T, O'Brien CA, Roberson PK, and Manolagas SC (1998). Transcriptional activation of the p21(WAF1, CIP1,SDI1) gene by interleukin-6 type cytokines. A prerequisite for their pro-differentiating and anti-apoptotic effects on human osteoblastic cells. J Biol Chem 273:21137-21144.

Bromberg JF, Wrzeszczynska MH, Devgan G, Zhao Y, Pestell RG, Albanese C, and Darnell JE, Jr (1999). Stat3 as an oncogene [published erratum appears in Cell 99:239]. Cell 98:295-303.

Chen RH, Chang MC, Su YH, Tsai YT, and Kuo ML (1999). Interleukin-6 inhibits transforming growth factor-betainduced apoptosis through the phosphatidylinositol 3-kinase/Akt and signal transducers and activators of transcription 3 pathways. J Biol Chem 274:23013-23019.

Chung TD, Yu JJ, Kong TA, Spiotto MT, and Lin JM (2000). Interleukin-6 activates phosphatidylinositol-3 kinase, which inhibits apoptosis in human prostate cancer cell lines. Prostate $42: 1-7$.

Du X, Liu Q, Yang Z, Orazi A, Rescorla FJ, Grosfeld JL, and Williams DA (1997). Protective effects of interleukin-11 in a murine model of ischemic bowel necrosis. Am $\mathrm{J}$ Physiol 272:G545-G552.

Fukada T, Hibi M, Yamanaka Y, Takahashi-Tezuka M, Fujitani Y, Yamaguchi T, Nakajima K, and Hirano T (1996). Two signals are necessary for cell proliferation induced by a cytokine receptor gp130: Involvement of STAT3 in antiapoptosis. Immunity 5:449-460.

Gimbrone MA Jr (1976). Culture of vascular endothelium. Prog Hemost Thromb 3:1-28.

Grossman D, McNiff JM, Li F, and Altieri DC (1999). Expression and targeting of the apoptosis inhibitor, survivin, in 
human melanoma [published erratum appears in $\mathrm{J}$ Invest Dermatol 114:609]. J Invest Dermatol 113:1076-1081.

Heinrich PC, Behrmann I, Muller-Newen G, Schaper F, and Graeve L (1998). Interleukin-6-type cytokine signalling through the gp130/Jak/STAT pathway. Biochem J 334:297314.

Hemmings BA (1997). Akt signaling: Linking membrane events to life and death decisions [comment]. Science 275: $628-630$.

Kaptein A, Paillard V, and Saunders M (1996). Dominant negative stat3 mutant inhibits interleukin-6-induced JakSTAT signal transduction. J Biol Chem 271:5961-5964.

Keith JC Jr, Albert L, Sonis ST, Pfeiffer CJ, and Schaub RG (1994). IL-11, a pleiotropic cytokine: Exciting new effects of IL-11 on gastrointestinal mucosal biology. Stem Cells Suppl $1: 79-89$.

Li F, Ackermann EJ, Bennett CF, Rothermel AL, Plescia J, Tognin S, Villa A, Marchisio PC, and Altieri DC (1999). Pleiotropic cell-division defects and apoptosis induced by interference with survivin function. Nat Cell Biol 1:461-466.

Mahboubi K, Biedermann BC, Carroll JM, and Pober JS (2000). IL-11 activates human endothelial cells to resist immune-mediated injury. J Immunol 164:3837-3846.

Mantell LL, Horowitz S, Davis JM, and Kazzaz JA (1999). Hyperoxia-induced cell death in the lung: The correlation of apoptosis, necrosis, and inflammation. Ann NY Acad Sci 887:171-180.

Murray AG, Schechner JS, Epperson DE, Sultan P, McNiff JM, Hughes CC, Lorber MI, Askenase PW, and Pober JS (1998). Dermal microvascular injury in the human peripheral blood lymphocyte reconstituted-severe combined immunodeficient (HuPBL-SCID) mouse/skin allograft model is T cell mediated and inhibited by a combination of cyclosporine and rapamycin. Am J Pathol 153:627-638.

O'Connor DS, Schechner JS, Adida C, Mesri M, Rothermel AL, Li F, Nath AK, Pober JS, and Altieri DC (2000). Control of apoptosis during angiogenesis by survivin expression in endothelial cells. Am J Pathol 156:1-6.

Oh H, Fujio Y, Kunisada K, Hirota H, Matsui H, Kishimoto T, and Yamauchi-Takihara K (1998). Activation of phosphatidylinositol 3-kinase through glycoprotein 130 induces protein kinase $B$ and p70 S6 kinase phosphorylation in cardiac myocytes. J Biol Chem 273:9703-9710.

Orazi A, Du X, Yang Z, Kashai M, and Williams DA (1996). Interleukin-11 prevents apoptosis and accelerates recovery of small intestinal mucosa in mice treated with combined chemotherapy and radiation. Lab Invest 75:33-42.
Papapetropoulos A, Fulton D, Mahboubi K, Kalb RG, O'Conner DS, Li F, Altieri DC, and Sessa WC (2000). Angiopoietin-1 inhibits endothelial cell apoptosis via the Akt/survivin pathway. J Biol Chem 275:9102-9105.

Qiu Y, Robinson D, Pretlow TG, and Kung HJ (1998). Etk/ $\mathrm{Bmx}$, a tyrosine kinase with a pleckstrin-homology domain, is an effector of phosphatidylinositol 3'-kinase and is involved in interleukin 6-induced neuroendocrine differentiation of prostate cancer cells. Proc Natl Acad Sci USA 95:36443649.

Ray P, Tang W, Wang P, Homer R, Kuhn C, Flavell RA, and Elias JA (1997). Regulated overexpression of interleukin 11 in the lung: Use to dissociate development-dependent and -independent phenotypes. J Clin Invest 100:2501-2511.

Reed JC and Reed SI (1999). Survivin' cell-separation anxiety. Nat Cell Biol 1:E199-E200.

Thornton SC, Mueller SN, and Levine EM (1983). Human endothelial cells: Use of heparin in cloning and long-term serial cultivation. Science 222:623-625.

Tran J, Rak J, Sheehan C, Saibil SD, LaCasse E, Korneluk $R G$, and Kerbel RS (1999). Marked induction of the IAP family antiapoptotic proteins survivin and XIAP by VEGF in vascular endothelial cells. Biochem Biophys Res Commun 264:781788.

Velculescu VE, Madden SL, Zhang L, Lash AE, Yu J, Rago C, Lal A, Wang CJ, Beaudry GA, Ciriello KM, Cook BP, Dufault MR, Ferguson AT, Gao Y, He TC, Hermeking H, Hiraldo SK, Hwang PM, Lopez MA, Luderer HF, Mathews B, Petroziello JM, Polyak K, Zawel L, and Kinzler KW (1999). Analysis of human transcriptomes [letter]. Nat Genet 23:387-388.

Walter MJ, Look DC, Tidwell RM, Roswit WT, and Holtzman MJ (1997). Targeted inhibition of interferon-gammadependent intercellular adhesion molecule-1 (ICAM-1) expression using dominant-negative Stat J Biol Chem 272: 28582-28589.

Waxman $A B$, Einarsson $O$, Seres $T$, Knickelbein RG, Warshaw JB, Johnston R, Homer RJ, and Elias JA (1998). Targeted lung expression of interleukin-11 enhances murine tolerance of $100 \%$ oxygen and diminishes hyperoxiainduced DNA fragmentation. J Clin Invest 101:1970-1982.

Zheng L, Dengler TJ, Kluger MS, Madge LA, Schechner JS, Maher SE, Pober JS, and Bothwell AL (2000). Cytoprotection of human umbilical vein endothelial cells against apoptosis and CTL-mediated lysis provided by caspase-resistant bcl-2 without alterations in growth or activation responses [In Process Citation]. J Immunol 164:4665-4671. 